\title{
Stevens-Johnson Syndrome (SJS) and Toxic Epidermal Necrolysis (TEN): an update
}

\author{
O Mogole' ${ }^{1}$ N Schellack ${ }^{1}$, MH Motswaledi ${ }^{2}$ \\ 'Department of Pharmacy, Faculty of Health Sciences, Sefako Makgatho Health Sciences University \\ ${ }^{2}$ Department of Dermatology, Faculty of Medicine, Sefako Makgatho Health Sciences University
}

Corresponding author, email: natalie.schellack@smu.ac.za

\begin{abstract}
Stevens-Johnson syndrome (SJS) is a form of toxic epidermal necrolysis (TEN) a rare but life-threatening hypersensitivity reactions that affect the skin and mucous membranes. The most common triggers are drugs, but they can also be triggered by infections. Granulysin has been recently identified as the major molecule responsible for the widespread keratinocyte necrosis. Early identification and removal of causative agent is crucial in preventing progression of condition and reducing patient mortality. Supportive care is often recommended over immunomodulating treatments as it helps improve patient outcome.
\end{abstract}

Keywords: Steven-Johnsons syndrome (SJS), toxic epidermal necrolysis (TEN), granulysin, supportive care, hypersensitivity, immunomodulating

\section{Introduction}

Stevens-Johnson syndrome (SJS) and toxic epidermal necrolysis (TEN) are delayed-type hypersensitivity reactions triggered by drugs, and sometimes by infections. ${ }^{1}$ They are characterised by widespread epidermal necrosis of the skin and mucosa and are associated with substantial morbidity. They are classified according to body surface area affected. ${ }^{2}$ SJS and TEN are typically severe and potentially fatal in the acute phase due to the necrosis of external and internal body surfaces which may predispose the patient to life-threatening complications such as sepsis and multi-organ failure. ${ }^{1}$ In South Africa, the majority of patients who present with SJS and TEN are young to middle aged women who are HIV positive, and the most commonly implicated drugs are nevirapine and co-trimoxazole. ${ }^{3}$

SJS and TEN rarely occur without any prior drug use, and in the rare instances that they do occur, the drug history reveals longterm medication use which cannot be linked directly to the adverse reaction experienced. ${ }^{4}$ Other instances where SJS and TEN have occurred include vaccination, exposure to industrial chemicals and fumes, consumption of natural remedies and traditional Chinese herbal medications, and also infections by Mycoplasma pneumoniae and the herpes simplex virus. ${ }^{5}$

\section{Pathophysiology}

Until recently, SJS and TEN was thought to be an idiopathic illness until research found that morbidity can result from a genetic predisposition to a drug hypersensitivity reaction. ${ }^{6}$ A cationic protein produced by cytotoxic $T$ lymphocytes and natural killer cells (NK cells), called granulysin, has been
Table I: Drugs that most commonly cause SJS and TEN

\begin{tabular}{ll} 
Class & Examples of drugs in the class \\
\hline Antibacterials & $\begin{array}{l}\text { Sulphonamides(trimethoprim/ } \\
\text { sulfamethoxazole), penicillins, tetracyclines, } \\
\text { quinolones, vancomycin }\end{array}$ \\
\hline Anticonvulsants & $\begin{array}{l}\text { Carbamazepine, phenytoin, phenobarbitone, } \\
\text { lamitrogine }\end{array}$ \\
\hline Antigout & Allopurinol \\
\hline Antimalarials & Chloroquine \\
\hline Antiretrovirals & Nevirapine, abacavir, protease inhibitors \\
Antituberculosis & Ethambutol, isoniazid \\
NSAIDS & Aspirin, piroxicam, diclofenac \\
\hline
\end{tabular}

recently identified as a major cytolytic molecule responsible for the widespread keratinocyte necrosis in SJS and TEN.? Granulysin is the most prevalent molecule found in SJS and TEN blisters and it is believed to act as a cytokine for destructive retinoid molecules such as retinoic acid. When the keratinocytes (which make up 90\% of the cells in the epidermis) die off, the epidermis detaches from the dermis resulting in tissue necrosis and sloughing. ${ }^{6}$ Mawson, Eriator, Karre 2016 simplifies the pathophysiology by hypothesising that the different medications implicated have a common property of interacting and synergising with endogenous retinoids, resulting in the accumulation and damage to the liver and induction of cholestatic liver dysfunction. This causes spillage of toxic retinoid compounds into circulation which extensively induces cytotoxicity and apoptosis via granulysin. 


\section{Clinical presentation}

SJS and TEN generally develop within the first eight weeks after starting a new medication. ${ }^{5}$ Initial symptoms include fever and malaise, followed by the development of a generalised, tender cutaneous eruption consisting of macules, papules, atypical target lesions, and vesicles or bullae. ${ }^{8}$ Mokenhaupt, (2016) further characterises these conditions by a cutaneous erythema with blister formation and haemorrhagic erosions of the mucous membranes such as stomatitis, balanitis, colpitis (vaginitis), severe conjunctivitis and blepharitis. The most widely accepted definition of these conditions is based on skin detachment, with SJS being defined when the denuded cutaneous surface covers less than $10 \%$ of the body surface area (figure 1) and TEN being defined when over $30 \%$ of the body surface is denuded (figure 2). ${ }^{9}$ Epidermal detachment between $10-30 \%$ constitutes SJS-TEN overlap. ${ }^{1}$

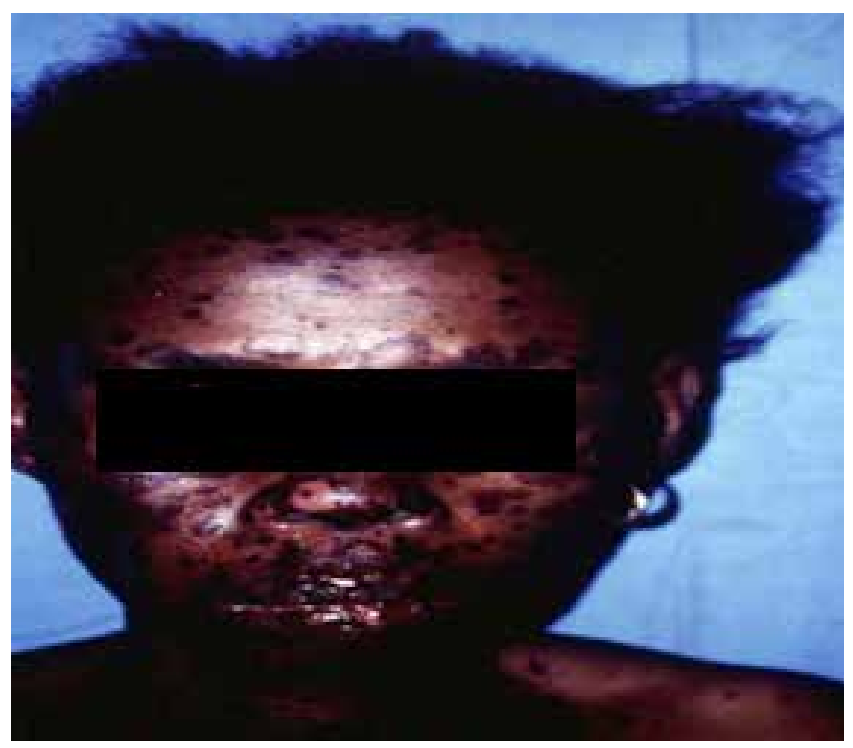

Figure 1: Stevens Johnson Syndrome (SJS): In a patient treated with Co-rimoxazole/Sulfamethoxazole

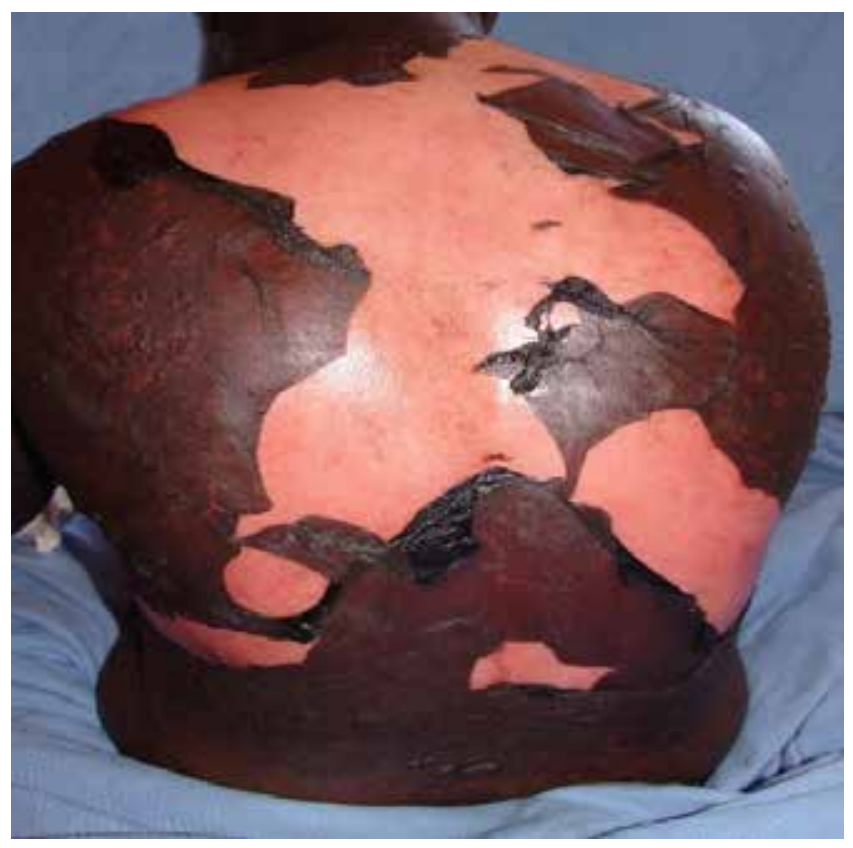

Figure 2: Toxic Epidermal Necrolysis due first line treatment for TB - note greater than $30 \%$ skin denudation

\section{Complications}

The most documented pulmonary complications of SJS are obstructive lung diseases which include bronchiolitis, bronchiectasis, and chronic obliterative bronchitis. These patients often present with a cough, dyspnoea, wheezing, purulent sputum and sometimes a fever. 'Gastrointestinal and hepatic complications that have been commonly documented are oesophageal strictures which usually appear one month after the onset of SJS/TEN, ' but Teo and Walsh ${ }^{10}$ (2016) also report that intestinal ulcers, chronic cholestasis, ischaemic hepatitis and the vanishing bile duct syndrome have also been documented.

Oral mucous membrane involvement is seen in the majority (70 - 100\%) of patients with SJS and TEN and includes tooth decay, severe dental growth abnormalities as a result of bacterial infection due to low saliva production and ulceration with fibrinous exudative inflammation which leads to reepithelialisation and healing. 'Some dental hypoplasia has also been documented in these patients. ${ }^{10}$ Otorhinolaryngologic involvement includes hypopharyngeal stenosis, nasal septal synechiae, pinna synechiae, ${ }^{10}$ acute laryngitis, epiglottitis, and ulceration of the nasal cavity which resolve with supportive therapy and airway management. ${ }^{1}$ Gynaecologic complications involve vulva and vaginal adenosis, a fusion of the labia minora and majora, and labial synechiae and renal complications involve glomerulonephritis and chronic renal insufficiency. ${ }^{10}$

\section{Management}

\section{Non-pharmacological treatment}

Early and aggressive supportive care isolated preferably in a Burns Intensive Care Unit may be one of the most important intervention for patients with SJS and TEN, and the offending medication should be identified and stopped immediately to improve prognosis. ${ }^{5}$ Room temperature should be increased to between 30 and 32 degrees Celsius, especially if a larger body surface area is involved and bedding on alternating pressure mattress is also recommended. Patients should receive fluid and electrolyte replacement and a solution of albumin ${ }^{4}$ to prevent end-organ hypoperfusion and shock. ${ }^{11}$ While the patient is in the ICU, necrotic tissue should be debrided and the exposed areas should be covered with an artificial membrane or a biological dressing to promote healing, reduced scarring and discomfort, and prevent infection. Due to the high rate of infection in patients with SJS and TEN, regular blood, skin and urine cultures should be performed. ${ }^{5}$

\section{Pharmacological treatment}

\section{Systemic corticosteroids}

Corticosteroids have been used in the management of SJS and TEN for many years despite high controversy about their use in these patients. Some literature emphasize that early highdose corticosteroids can be used to inhibit inflammation, ${ }^{11}$ while some literature state that corticosteroids increase the rate of infections, the risk of masking septicaemia and also delay re-epithelialisation which ultimately prolongs hospital duration and increases mortality. ${ }^{4} \mathrm{~A}$ high dose of pulsed 
intravenous dexamethasone (1.5mg/kg/day), when given for three days, seems to stop disease progression and promote healing within three weeks. ${ }^{5}$

\section{Human intravenous immunoglobulin (IVIg)}

Human intravenous immune globulin (IVIg) has been used for both treatment and prophylaxis of SJS and TEN because it has been hypothesised that antibodies in pooled human IVIg inhibit Fas-mediated keratinocyte necrosis. ${ }^{4}$ Creamer et al 2016 showed that mortality in patients using IVIg showed no improvement as compared to patients receiving supportive care alone, while Mokwnhaupt (2016) concluded that IVIg is not the best treatment for SJS and TEN.

\section{Cyclosporine}

Cyclosporine inhibits apoptosis through the inhibition of down regulation of NF-kB and has reported good results when given at a dose of $3 \mathrm{mg} / \mathrm{kg} /$ day for ten days. ${ }^{5}$ Cyclosporine is used due to its speculated effects on granulysin and its reduction on mortality rate. ${ }^{4}$

\section{Plasmapheresis}

Plasmapheresis is the process where non-dialyzable pathogenic components are removed from plasma. The blood is then reconstituted by adding albumin to artificial plasma and re-infused back into the patient. ${ }^{5}$

\section{Cyclophosphamide}

The benefits of cyclophosphamide in patients with acute TEN was previously reported but is not recommended anymore in the treatment of SJS and TEN due an associated increase in mortality rate. ${ }^{5}$

\section{TNF-alpha inhibitors}

Infliximab, pentoxiphylline and thalidomide are used in the management of SJS and TEN due to their inhibition of TNFalpha which is said to play a role in SJS and TEN. ${ }^{5}$

\section{Granulocyte colony stimulating factor}

Granulocyte colony stimulating factor is used to boost neutrophil count in patients with SJS and TEN to reduce the risk of infection caused by neutropenia. ${ }^{5}$

\section{Conclusion}

SJS and TEN are severe; T-cell mediated drug reactions which result in rapid irreversible keratinocyte cell death. Although SJS is less severe than TEN, they both have the same aetiology, genetic susceptibility and pathogenesis. Patients most at risk include those taking; allopurinol, sulphonamide anti-infectives (especially co-trimoxazole), carbamezapine, phenytoin, phenobarbital and non-steroidal anti-inflammatory medicines. Due to the rarity of the disease, direct evidence is difficult to mention on any specific treatment, but supportive care in an intensive care or specialist burn unit has been found to be the best management of patients with SJS and TEN. The standard treatment includes analgesia, wound care, fluid replacement, nutritional support and infection control. The benefits of using high-dose corticosteroids, IVIg and plasmapheresis have not yet proven to be effective and therefore further large, registry-based retrospective studies are needed to improve the management of SJS and TEN. The sequelae of the disease is very difficult to predict, so therefore patients should be monitored and followed up for signs of complications after the resolution of the acute phase and also be educated on avoiding the causative medication.

\section{References}

1. Saeed H, Mantagos IS, Chodosh J. Complications of Stevens-Johnson syndrome beyond the eye and skin. Burns. 2016 Feb 29;42(1):20-7. [Accessed 11 August 2016]. http://dx.doi.org/10.1016/j.burns.2015.03.012

2. Hsu DY, Brieva J, Silverberg NB, Silverberg J. Morbidity and Mortality of StevensJohnson Syndrome and Toxic Epidermal Necrolysis in United States Adults. Journal of Investigative Dermatology. 2016 Mar 30. [Accessed 1 August 2016]. http://dx.doi.org.10.1016.j.jid.2016.03.023

3. Kannenberg SM, Jordaan HF, Koegelenberg CF, Von Groote-Bidlingmaier F, Visser WI. Toxic epidermal necrolysis and Stevens-Johnson syndrome in South Africa: a 3-year prospective study. QJM. 2012 Sep 1;105(9):839-46. [Accessed11 September 2016]. Doi:10.1093/qjmed/hcs078

4. Mockenhaupt, M. "The Current Understanding Of Stevens-Johnson Syndrome And Toxic Epidermal Necrolysis". Expert Review of Clinical Immunology 7.6 (2011): 803-815. [Accessed 1 August 2016]. http://dx.doi.org/10.1586/eci.11.66

5. Kohanim S, Palioura S, Saeed HN, Akpek EK, Amescua G, Basu S, Blomquist PH, Bouchard CS, Dart JK, Gai X, Gomes JA. Stevens-Johnson Syndrome/ Toxic Epidermal Necrolysis-A Comprehensive Review and Guide to Therapy. I. Systemic Disease. The ocular surface. 2016 Jan 31;14(1):2-19. [Accessed 6 August 2016]. http://dx.doi.org.10.1016/j.jtos.2015.10.002

6. Klein DM. Pathophysiology-Stevens-Johnson Syndrome/Toxic Epidermal Necrolysis. (2016). [Accessed 10 August 2016].

7. Mawson AR, Eriator I, Karre S. Stevens-Johnson syndrome and toxic epidermal necrolysis (SJS/TEN): could retinoids play a causative role?. Medical Science Monitor. 2015 Jan 12;21:133-43. [Accessed 10 August 2016]. Doi:10.12659/ MSM.891043

8. Miliszewski MA, Kirchhof MG, Sikora S, Papp A, Dutz JP. Stevens-Johnson Syndrome and Toxic Epidermal Necrolysis: An Analysis of Triggers and Implications for Improving Prevention. The American journal of medicine. 2016 Apr 15. [Accessed 1 August 2016]. http://dz.doi. org10.1016.j.amjmed.2016.03.022

9. Jain R, Sharma N, Basu S, lyer G, Ueta M, Sotozono C, Kannabiran C, Rathi VM, Gupta N, Kinoshita S, Gomes JA. Stevens-Johnson syndrome: The role of an ophthalmologist. Survey of ophthalmology. 2016 Jan 30. [Accessed 1 August 2016]. http://dx.doi.org./10.1016/j.survophthal.2016.01.004

10. Teo YX, Walsh SA. Severe adverse drug reactions. Clinical Medicine. 2016 Feb 1;16(1):79-83. [Accessed 9 August 2016]. Doi: 10.7861/clinmedicine.16-1-79

11. Creamer D, Walsh SA, Dziewulski P, Exton LS, Lee HY, Dart JK, Setterfield J, Bunker $\mathrm{CB}$, Ardern-Jones MR, Watson KM, Wong GA. UK guidelines for the management of Stevens-Johnson syndrome/toxic epidermal necrolysis in adults 2016. Journal of Plastic, Reconstructive \& Aesthetic Surgery. 2016 Jun 30;69(6):e119-53. [Accessed 8 August 2016]. http:dx.doi.org.10.1016.j.bips.2016.01.034 